\title{
A Breakdown Zone Model of a Fault in a Strip
}

\author{
Takuo Maruyama ${ }^{1, *}$ and Tian-You Fan ${ }^{2}$ \\ ${ }^{1}$ Earthquake Research Institute, The University of Tokyo, \\ Bunkyo-ku, Tokyo 113, Japan \\ ${ }^{2}$ Research Centre of Materials Science, Beijing Institute of Technology, \\ P.O. Box 327, Beijing 100081, China
}

\begin{abstract}
A breakdown zone model for a fault of length $a$ on $y=0$ is constructed in a strip of width $2 \mathrm{H}$, based on two-dimensional elasticity theory. The three-dimensional concept from which this model is derived consists in that a strike slip fault exists in a thin brittle regime overlying a ductile regime or asthenosphere, and that outside of the strip in the brittle regime stresses or displacements will be changed little in the formation of a new shear crack surface. Two types of boundary condition are considered in an idealized form, i.e., zero stress change and zero displacement change on the boundary $y= \pm H$. To obtain the stress intensity factor for a semi-infinite crack in a strip, numerical methods are developed for solving integral equations. The results show that the critical shear stress against the ratio $a / H$ for the critical state of fault formation separates in two branches at the point $a / H=0$, according to those two types of boundary condition.

What is relevant to our three-dimensional concept of a strike slip fault lies between the two cases. Then an expression for the critical shear stress is suggested, to a first approximation, independent of $a / H$. This is the same with the formula for an ordinary breakdown zone model in a homogeneous infinite medium. Since in our case, however, material constants are taken in the strip, our model may serve as a breakdown zone model of a strike slip fault related to a structural weak line in the crust.
\end{abstract}

\section{Introduction}

Slip-weaking instability models for a fault (cf., Rice, 1980) have been developed as an extension to shear faulting by Palmer and Rice (1973), Ida (1972) and others of the cohesive zone models of tensile fracture by Barenblatt (1962). Up to now most studies in this direction are limited to the analyses for a fault in a homogeneous and infinite region.

In Fig. 1(a) a homogeneous medium under uniform shear stress is schematically depicted. As in a usual treatment of two-dimensional elasticity theory, the stress in the medium is being produced by forces acting on the boundary; if we remove them, the medium comes to the state of zero stress. Though a shear crack considered in such configuration is simple and fundamental, it seems insufficient for a model of actual

Received September 2, 1992; Accepted February 2, 1993

* To whom correspondence should be addressed. 
(a)

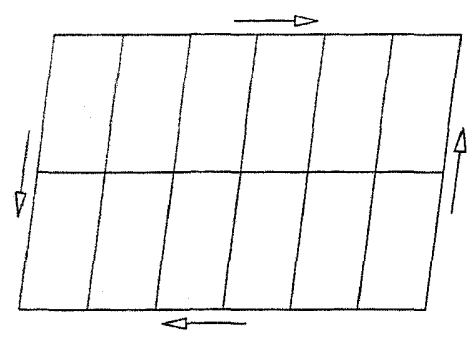

(b)

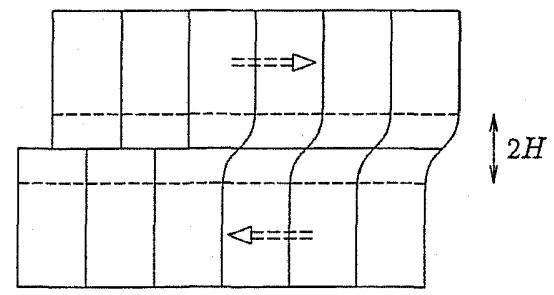

Fig. 1. (a) A homogeneous medium under shear stresses. (b) A schematic model of a fault in the brittle regime overlying the ductile regime in non-uniform movements shown by broken arrows.

strike slip faults in the crust from geophysical point of view. We can pick up at least two reasons. (1) In a homogeneous shear stress state as in Fig. 1(a), two directions of maximum shear exist and they are equally effective in reducing shear stress in the medium. But in big faults in nature one direction is preferred. This shows that we should have a model that is adapted for a structural weak line, or such inhomogeneity of the medium. (2) In the case of strike slip faults, though the origin of stress may partly be the forces acting on the remote boundary of the block, main causes of stress in the crust will originate in the inhomogeneity in the movement of underlying ductile regime. For a thin plate, forces acting on the base surface will be much more effective than those on the boundary.

We then adopt a model as depicted in Fig. 1(b), in which arrows in broken line correspond to non-uniform movements of the underlying ductile regime or asthenosphere. As we employ static elasticity theory in the case, the stress change in the formation of a new fault surface is considered to propagate in an infinite speed. In such a situation the part at a distance from the fault may play as a standstill for a short time interval of the formation of a new crack surface.

Thus we have a model of a fault within a narrow region of width $2 H$ in which large strains are cofined. As to the boundary condition on the boundary $y= \pm H$ in the formation of a new fault surface of length $a$ on $y=0$, we consider two cases in an idealized form:

(i) the stresses have not changed, (ii) the displacements have not changed.

We regard the model as a two-dimensionally simplified model of a strike slip fault in a brittle regime overlying a ductile regime that moves with non-uniform speed. From 
our concept for the model of fault, $2 \mathrm{H}$ does not directly correspond to the thickness of the fault, but to an effective width in which large strains are confined.

As for a strip under zero displacement change boundary conditions, for $a / H \rightarrow \infty$, the stress intensity factor for a crack has been derived, for plane strain, in energy release rate argument (or $J$-integral result) as

$$
K_{I I}=\sqrt{\frac{2}{1-\nu}}\left(\tau-\tau_{f}\right) \sqrt{H},
$$

where $v$ is Poisson's ratio, $\tau$ is the applied shear stress and $\tau_{f}$ is the frictional shear stress (Palmer and Rice, 1973). Since, in this case, $K_{I I}$ is independent of fault length $a$, we cannot construct any breakdown zone model. We shall examine the case where the ratio $a / H$ is smaller.

Under zero stress change boundary conditions, Fan (1990) offered an analytical expression for the stress intensity factor for a semi-infinite crack in a strip, by introducing a conformal mapping technique. In the following, this mapping will be developed in numerical calculations for the two types of boundary value problems.

\section{Fundamental Analyses}

In Fig. 1(b) $H$ stands for the half-width of the strip confining large strains, and the fault has a semi-infinite length. The stress on the fault surface is considered to be equivalent to one which without slipping would result in a shear stress of magnitude $\tau$ on the fault plane for a distance $a$ extending back from the fault tip, and of magnitude $\tau_{f}$ over the remainder of the fault. The size $a$ is taken to simulate a fault of finite length. With the frictional shear stress $\tau_{f}$ on the fault face, the total decreased shear stress is $\left(\tau-\tau_{f}\right)$ over distance $a$, and vanishing over the remainder. Near the fault tip, there is a so-called breakdown zone with length $R$ whose value is unknown a priori. In this zone, a reverse shear stress drop $\left(\tau_{b}-\tau_{f}\right)$ is effected as shown in Fig. 2 (cf., Rice and Simons, 1976), where $\tau_{b}$ represents the breakdown limit, a material constant.

According to the plane elasticity theory (Muskhelishvili, 1953), stresses $\sigma_{x x}, \sigma_{x y}$, $\sigma_{y y}$, and displacements $u_{x}, u_{y}$ can be expressed by means of two analytic functions $\phi_{1}(z)$ and $\psi_{1}(z)$ of $z=x+i y$ as

$$
\left.\begin{array}{l}
\sigma_{x x}+\sigma_{y y}=2\left[\phi_{1}^{\prime}(z)+\overline{\phi_{1}^{\prime}(z)}\right] \\
\sigma_{y y}-\sigma_{x x}+2 i \sigma_{x y}=2\left[\bar{z} \phi_{1}^{\prime \prime}(z)+\psi_{1}^{\prime}(z)\right], \\
2 \mu\left(u_{x}+i u_{y}\right)=\kappa \phi_{1}(z)-z \overline{\phi_{1}^{\prime}(z)}-\overline{\psi_{1}(z)},
\end{array}\right\}
$$

where $\bar{z}, \overline{\phi_{1}(z)}$, etc. denote the complex conjugates of $z, \phi_{1}(z)$, etc., respectively, $\phi_{1}^{\prime}(z)=\mathrm{d} \phi_{1} / \mathrm{d} z, \psi_{1}^{\prime}(z)=\mathrm{d} \psi_{1}^{\prime} / \mathrm{d} z$, and

$$
\kappa= \begin{cases}3-4 v, & \text { (plane strain) } \\ (3-v) /(1+v), & \text { (plane stress) }\end{cases}
$$

where parameters $\mu$ and $v$ are the shear modulus and the Poisson's ratio of the material, respectively.

Vol. 41, No. 1, 1993 


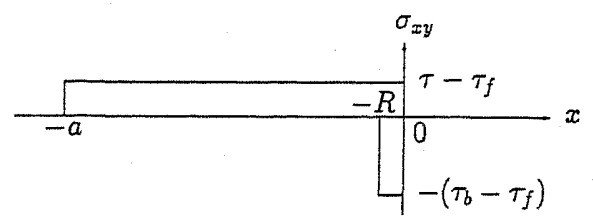

Fig. 2. Assumed stresses near the fault tip for $-a<x<0$ and $-R<x<0$.

If we take an $\operatorname{arc} A B$ in the region, let its positive direction be from $A$ to $B$ and draw the normal $n$ to the right of the arc when looking along it in the positive direction, then we have

$$
\left[\phi_{1}(z)+z \overline{\phi_{1}^{\prime}(z)}+\overline{\psi_{1}(z)}\right]_{A}^{B}=i \int_{A B}\left(\stackrel{n}{T_{x}}+i \stackrel{n}{T_{y}}\right) \mathrm{d} s,
$$

where $\left(\stackrel{n}{T}_{x} \mathrm{~d} s, \stackrel{n}{T}_{y} \mathrm{~d} s\right)$ is the force across an element $\mathrm{d} s$ of the arc $A B$, exerted by the positive side of normal upon the negative side.

\subsection{Case of zero stress change on the boundary $y= \pm H$}

The problem corresponding to the decreased stress $\left(\tau-\tau_{f}\right)$ on $y=0$ and no stress change on the boundary $y= \pm H$ has the following boundary conditions:

$$
\left.\begin{array}{ll}
y= \pm H,-\infty<x<\infty: & \sigma_{y y}=\sigma_{x y}=0, \\
x= \pm \infty,-H<y<H: & \sigma_{x x}=\sigma_{x y}=0, \\
y= \pm 0,-\infty<x<-a: & \sigma_{y y}=\sigma_{x y}=0, \\
y= \pm 0,-a<x<0 \quad: & \sigma_{y y}=0, \quad \sigma_{x y}=-\left(\tau-\tau_{f}\right) .
\end{array}\right\}
$$

We take the region as a strip cut along the negative $x$-axis and consider a contour $L$ surrounding the region in the counter-clockwise direction (Fig. 3(a)), starting at some fixed point $z_{A}$ on the boundary. Then since in Eq. (4)

$$
\begin{gathered}
\quad \stackrel{n}{T_{x}=\tau-\tau_{f},} \quad \stackrel{n}{T_{y}=0,} \mathrm{~d} s=\mathrm{d} x, \quad \text { for } \quad-a<x<0, \quad y=+0, \\
n_{x}^{n}=-\left(\tau-\tau_{f}\right), \quad \stackrel{n}{T_{y}=0,} \mathrm{~d} s=-\mathrm{d} x, \quad \text { for }-a<x<0, \quad y=-0,
\end{gathered}
$$

we have, for an arbitrary point $z$ on $L$,

$$
\phi_{1}(z)+z \overline{\phi_{1}^{\prime}(z)}+\overline{\psi_{1}(z)}=f_{1}(z)+\text { const },
$$

where

$$
\left.f_{1}(z)=i \int_{z_{A}}^{z} \stackrel{n}{T_{x}}+i \stackrel{n}{T}_{y}\right) \mathrm{d} s= \begin{cases}i\left(\tau-\tau_{f}\right)(z+a), & (-a<x<0, y= \pm 0) \\ 0, & \text { (for other } z \text { on } L)\end{cases}
$$

we take constant in Eq. (6) to be zero.

To solve the problem, Fan (1990) suggested the conformal mapping

$$
z=\omega(\zeta)=\frac{H}{\pi} \ln \left[1+\left(\frac{1+\zeta}{1-\zeta}\right)^{2}\right]
$$


(a)

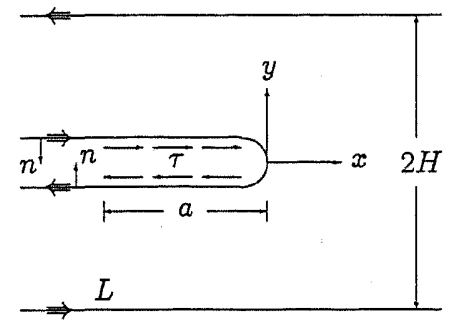

(b)

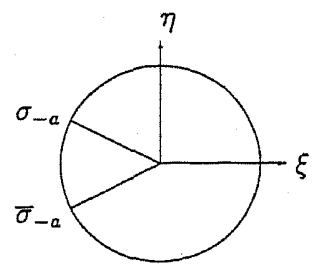

Fig. 3. (a) Contour line $L$ surrounding the cracked strip. (b) Unit circle $\gamma$ in $\zeta(=\xi+i \eta)$ plane.

to transform the region shown in Fig. 3(a) onto a unit circle $\gamma$ in $\zeta(=\xi+i \eta)$ plane shown in Fig. 3(b). By this mapping, Eq. (6) becomes

$$
\phi(\sigma)+\omega(\sigma) \frac{\overline{\phi^{\prime}(\sigma)}}{\omega^{\prime}(\sigma)}+\overline{\psi(\sigma)}=f(\sigma)
$$

where $\sigma=\left.\zeta\right|_{\gamma}=\exp (i \theta)$ represents the value of $\zeta$ on the unit circle $\gamma$ and we used the following notations:

$$
\left.\begin{array}{l}
\phi_{1}(z)=\phi_{1}(\omega(\zeta))=\phi(\zeta) ; \quad \psi_{1}(z)=\psi_{1}(\omega(\zeta))=\psi(\zeta) ; \\
\phi_{1}^{\prime}(z)=\phi^{\prime}(\zeta) / \omega^{\prime}(\zeta) ; \quad \psi_{1}^{\prime}(z)=\psi^{\prime}(\zeta) / \omega^{\prime}(\zeta) ; \\
f_{1}(z)=f_{1}(z(\sigma))=f(\sigma), \quad \text { for } z \text { on } L .
\end{array}\right\}
$$

Since the stress intensity factors are related to $\phi^{\prime}(\zeta)$ by the relation

$$
K_{I}-i K_{I I}=2 \sqrt{2 \pi} \lim _{z \rightarrow 0} \sqrt{z} \phi_{1}^{\prime}(z)=2 \sqrt{\pi} \lim _{\zeta \rightarrow-1} \frac{\phi^{\prime}(\zeta)}{\sqrt{\omega^{\prime \prime}(\zeta)}},
$$

we seek to find $\lim _{\zeta \rightarrow-1} \phi^{\prime}(\zeta)$. From Eq. (8), we have

$$
\omega^{\prime \prime}(-1)=\frac{H}{2 \pi} \text {. }
$$

In Eq. (9) we should consider $\omega(\sigma)$ as the boundary value of $\omega(\zeta)$ from inside $\gamma$ where $\omega(\zeta)$ denotes a branch that is regular inside $\gamma$. To note the connection with the branch that is regular outside $\gamma$ we may write $\omega(\zeta)$ as

$$
\begin{aligned}
\omega(\zeta) & =\frac{H}{\pi}[\ln (i-\zeta)+\ln (-i-\zeta)-2 \ln (1-\zeta)+\ln 2], \\
& =\frac{H}{\pi}\left(\int_{1}^{i} \frac{1}{\sigma-\zeta} \mathrm{d} \sigma-\int_{-i}^{1} \frac{1}{\sigma-\zeta} \mathrm{d} \sigma+\ln 2\right) .
\end{aligned}
$$

This shows that if we consider two branches that are regular inside and outside $\gamma$ at the same time, they have a discontinuity $(H / \pi) 2 \pi i$ on the arc starting at $\zeta=1$ and ending

Vol. 41, No. 1, 1993 

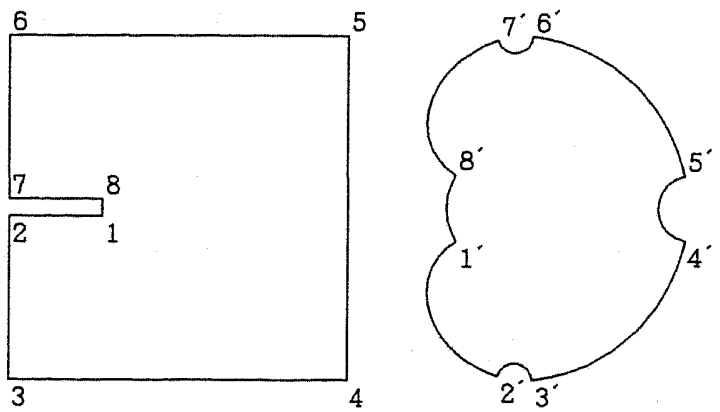

Fig. 4. A square with its two parallel sides on $y= \pm H$ and with a slit of finite width along the negative $x$-axis (left) and a representation of conformal transformation of the square by $\omega(\zeta)$ (right).

at $\zeta=i$, an opposite discontinuity $-(H / \pi) 2 \pi i$ on the arc starting at $\zeta=-i$ and ending at $\zeta=1$. To illustrate the mapping by $\omega(\zeta)$, an example of a square with its two parallel sides on $y= \pm H$ and with a slit of finite width along the negative $x$-axis is shown on the left and a representation of transformed region on the right, respectively, in Fig. 4. The vertices named as $1,2, \cdots, 8$ correspond to those named as $1^{\prime}, 2^{\prime}, \cdots, 8^{\prime}$, respectively. Though the transformed region by mapping in this case has a worm-eaten boundary, we see that as the length of strip becomes longer indefinitely and as the slit tends to a semi-infinite line, the rugged boundary tends to a complete unit circle.

Having numerical calculations in mind, we notice that the second term on the left sides of Eq. (9) can be written as

$$
[\omega(\sigma)-\overline{\omega(\sigma)}] \frac{\overline{\phi^{\prime}(\sigma)}}{\overline{\omega^{\prime}(\sigma)}}+\overline{\omega(\sigma)} \frac{\overline{\phi^{\prime}(\sigma)}}{\overline{\omega^{\prime}(\sigma)}},
$$

where as seen from the mapping by Eq. (8) and in the illustration of Fig. 4, we have, on $\gamma$,

$$
\omega(\sigma)-\overline{\omega(\sigma)}= \begin{cases}\left(\frac{H}{\pi}\right) 2 \pi i, & \left(0<\theta<\frac{\pi}{2}\right) \\ 0, & \left(\frac{\pi}{2}<\theta<\frac{3 \pi}{2}\right) \\ -\left(\frac{H}{\pi}\right) 2 \pi i . & \left(\frac{3 \pi}{2}<\theta<2 \pi\right)\end{cases}
$$

Hereafter the part $(\pi / 2 \leq \theta<3 \pi / 2)$ of $\gamma$ is denoted by $\gamma_{L}$, while the part $(-\pi / 2 \leq \theta<\pi / 2)$ of $\gamma$ by $\gamma_{R}$.

Dividing each of Eq. (9) by $2 \pi i(\sigma-\zeta)$ with $\zeta$ inside $\gamma$, we integrate its both sides with respect to $\sigma$ on $\gamma$, and we obtain

$$
\phi(\zeta)+I_{p}(\zeta)+\text { const }=F(\zeta)
$$

where $I_{p}(\zeta)$ is 


$$
I_{p}(\zeta)=\left(\int_{1}^{i}-\int_{-i}^{1}\right) \frac{(\sigma-1)\left(\sigma^{2}+1\right)}{2(\sigma+1) \sigma^{2}} \frac{\overline{\phi^{\prime}(\sigma)}}{\sigma-\zeta} \mathrm{d} \sigma
$$

by Eq. (12) and

$$
F(\zeta)=\frac{1}{2 \pi i} \int_{\gamma} \frac{f(\sigma)}{\sigma-\zeta} \mathrm{d} \sigma
$$

In obtaining Eq. (13), we considered a theorem referred later before Eq. (27), and in moving from Eqs. (9) to (13), the explicit expression

$$
\frac{\omega(\zeta)}{\omega^{\prime}(\zeta)}=\frac{(1-\zeta)\left(1+\zeta^{2}\right)}{2(1+\zeta)} \ln \left[1+\left(\frac{1+\zeta}{1-\zeta}\right)^{2}\right] .
$$

So far we have assumed that the function $\omega(\zeta) \phi^{\prime}(\zeta) / \omega^{\prime}(\zeta)=z \phi_{1}^{\prime}(z)$ as well as $\phi(\zeta)=\phi_{1}(z)$ and $\psi(\zeta)=\psi_{1}(z)$ are considered to be regular inside $\gamma$, continuous up to its boundary. If we pay attention to the fact that the function $\phi^{\prime}(\zeta)$ may have poles at $\zeta= \pm i$ or $\zeta=1$, as seen in the relation

$$
\phi_{1}^{\prime}(z)=\frac{\phi^{\prime}(\zeta)}{\omega^{\prime}(\zeta)}=\frac{\pi}{H} \frac{(1-\zeta)\left(1+\zeta^{2}\right)}{2(1+\zeta)} \phi^{\prime}(\zeta),
$$

we should introduce a new function $h(\zeta)$ defined by

$$
h(\zeta)=(1-\zeta)\left(1+\zeta^{2}\right) \phi^{\prime}(\zeta) .
$$

The function $h(\zeta)$ is considered to be regular inside $\gamma$, continuous up to its boundary.

Equation (13) may then be justified as considered to be the limit of $\varepsilon \rightarrow 0$, where $\varepsilon$ is small radius of the semi-circles, about the points $\zeta= \pm i$, of the rugged boundary, as shown in Fig. 4. Differentiation of Eq. (13) with respect to $\zeta$, with multiplication by $(1-\zeta)\left(1+\zeta^{2}\right)$, yields

$$
h(\zeta)+(1-\zeta)\left(1+\zeta^{2}\right) I_{p}^{\prime}(\zeta)=(1-\zeta)\left(1+\zeta^{2}\right) F^{\prime}(\zeta)
$$

An explicit expression of $F^{\prime}(\zeta)$ is given by

$$
\begin{aligned}
F^{\prime}(\zeta)= & \frac{\left(\tau-\tau_{f}\right)}{2 \pi} \frac{H}{\pi}\left\{\frac{2}{1-\zeta}[\ln (\sigma-\zeta)-\ln (\sigma-1)]\right. \\
& \left.+\frac{1}{1+\zeta^{2}}[2 \zeta \ln (\sigma-\zeta)-(\zeta-i) \ln (\sigma+i)-(\zeta+i) \ln (\sigma-i)]\right\}\left.\right|_{\sigma=\sigma_{-a}} ^{\bar{\sigma}_{-a}},
\end{aligned}
$$

where

$$
\sigma_{-a}=\frac{-\exp (-\pi a / H)+2 i \sqrt{1-\exp (-\pi a / H)}}{2-\exp (-\pi a / H)} .
$$

In Eq. (19) we see that $F^{\prime}(\zeta)$ has a pole neither at $\zeta= \pm i$ nor at $\zeta=1$ though it has a logarithmic singularity at $\zeta=\sigma_{-a}$ and at $\zeta=\bar{\sigma}_{-a}$. 


\subsection{Case of zero displacement change on the boundary $y= \pm H$}

The mixed boundary value problem corresponding to the decreased stress $\left(\tau-\tau_{f}\right)$ on $y=0$ while the boundary $y= \pm H$ is fixed has the following boundary conditions:

$$
\left.\begin{array}{ll}
y= \pm H,-\infty<x<\infty: & u_{x}=u_{y}=0 \\
x= \pm \infty,-H<y<H: & u_{x}=u_{y}=0, \\
y= \pm 0,-\infty<x<-a: & \sigma_{y y}=\sigma_{x y}=0 \\
y= \pm 0,-a<x<0 \quad: & \sigma_{y y}=0, \sigma_{x y}=-\left(\tau-\tau_{f}\right) .
\end{array}\right\}
$$

Owing to Eq. (1) and the mapping by Eq. (8), the conditions can be written in terms of $\phi(\zeta)$ and $\psi(\zeta)$ as

$$
\left.\begin{array}{ll}
\phi(\sigma)+\omega(\sigma) \frac{\overline{\phi^{\prime}(\sigma)}}{\overline{\omega^{\prime}(\sigma)}}+\overline{\psi(\sigma)}=f(\sigma)+c, & \left(\text { for } \sigma \text { on } \gamma_{L}\right) \\
-\kappa \phi(\sigma)+\omega(\sigma) \frac{\overline{\phi^{\prime}(\sigma)}}{\overline{\omega^{\prime}(\sigma)}}+\overline{\psi(\sigma)}=0, & \left(\text { for } \sigma \text { on } \gamma_{R}\right)
\end{array}\right\}
$$

where in the upper formula, constant in Eq. (6) is retained as $c$. By putting $\sigma= \pm i$ in Eq. (22), since $\left.f(\sigma)\right|_{\sigma= \pm i}=0$, we find

$$
c=(1+\kappa) \phi( \pm i) \text {. }
$$

Dividing each of Eq. (22) by $2 \pi i(\sigma-\zeta)$ with $\zeta$ inside $\gamma$, we obtain by integration with respect to $\sigma$ on $\gamma_{L}$ and on $\gamma_{R}$ and by addition

$$
\phi(\zeta)-\frac{1+\kappa}{2 \pi i} \int_{\gamma_{\mathrm{R}}} \frac{\phi(\sigma)}{\sigma-\zeta} \mathrm{d} \sigma+I_{p}(\zeta)+\text { const }=F(\zeta)+\frac{c}{2 \pi i} \ln \left(\frac{\zeta+i}{\zeta-i}\right),
$$

with $I_{p}(\zeta)$ and $F(\zeta)$ as before. We notice here that in Eq. (15), $f(\sigma)=0$ on $\sigma_{R}$. Strictly the above equation may be obtained as the limit as $\varepsilon \rightarrow 0$, where $\varepsilon$ is the radius of the semi-circles of the rugged boundary about $\zeta= \pm i$ as before. Differentiation of the equation with respect to $\zeta$, with $c$ in Eq. (23), yields

$$
\phi^{\prime}(\zeta)-\frac{1+\kappa}{2 \pi i} \int_{\gamma_{R}} \frac{\phi^{\prime}(\sigma)}{\sigma-\zeta} \mathrm{d} \sigma+I_{p}^{\prime}(\zeta)=F^{\prime}(\zeta)
$$

To apply Cauchy's integral formula to this equation we move to $h(\zeta)$ as defined in Eq. (17) by multiplication by $(1-\zeta)\left(1+\zeta^{2}\right)$ and obtain

$$
\begin{aligned}
h(\zeta)-\frac{1+\kappa}{2 \pi i}\left\{\int_{\gamma_{R}} \frac{h(\sigma)}{\sigma-\zeta} \mathrm{d} \sigma\right. & \left.-(1-\zeta) \int_{\gamma_{R}} \sigma \phi^{\prime}(\sigma) \mathrm{d} \sigma+\int_{\gamma_{R}} \sigma^{2} \phi^{\prime}(\sigma) \mathrm{d} \sigma\right\} \\
& +(1-\zeta)\left(1+\zeta^{2}\right) I_{p}^{\prime}(\zeta)=(1-\zeta)\left(1+\zeta^{2}\right) F^{\prime}(\zeta) .
\end{aligned}
$$

In obtaining the term in the braces, we have proceeded as follows. In an equation valid for any $\sigma$ and $\zeta$, 


$$
\begin{aligned}
& \frac{(1-\zeta)\left(1+\zeta^{2}\right)}{(1-\sigma)\left(1+\sigma^{2}\right)} \frac{h(\sigma)}{(\sigma-\zeta)}=\frac{h(\sigma)}{\sigma-\zeta} \\
& \quad+\frac{1}{2}\left[\left(1+\zeta^{2}\right) \frac{h(\sigma)}{1-\sigma}-(1-\zeta)(1+\zeta) \frac{\sigma h(\sigma)}{1+\sigma^{2}}+(1-\zeta)^{2} \frac{h(\sigma)}{1+\sigma^{2}}\right]
\end{aligned}
$$

expressing $h(\sigma)$ in terms of $\phi^{\prime}(\sigma)$ by Eq. (17), we have

$$
\begin{aligned}
& \int \frac{(1-\zeta)\left(1+\zeta^{2}\right)}{(1-\sigma)\left(1+\sigma^{2}\right)} \frac{h(\sigma)}{(\sigma-\zeta)} \mathrm{d} \sigma=\int \frac{h(\sigma)}{\sigma-\zeta} \mathrm{d} \sigma \\
& \quad+\left(1-\zeta+\zeta^{2}\right) \int \phi^{\prime}(\sigma) \mathrm{d} \sigma-(1-\zeta) \int \phi^{\prime}(\sigma) \sigma \mathrm{d} \sigma+\int \phi^{\prime}(\sigma) \sigma^{2} \mathrm{~d} \sigma
\end{aligned}
$$

and then applied it to $\gamma_{R}$ and considered that

$$
\int_{\gamma_{\mathbf{R}}} \phi^{\prime}(\sigma) \mathrm{d} \sigma=\phi(i)-\phi(-i)=0 .
$$

Using the relations

$$
\begin{aligned}
& \int_{\gamma_{R}} \phi^{\prime}(\sigma) \sigma \mathrm{d} \sigma=-\int_{\gamma_{L}} \phi^{\prime}(\sigma) \sigma \mathrm{d} \sigma, \\
& \int_{\gamma_{R}} \phi^{\prime}(\sigma) \sigma^{2} \mathrm{~d} \sigma=-\int_{\gamma_{L}} \phi^{\prime}(\sigma) \sigma^{2} \mathrm{~d} \sigma,
\end{aligned}
$$

in Eq. (24), we arrive at an integral equation

$$
\begin{aligned}
\int_{1}^{i}(- & \left.\frac{\kappa}{2 \pi i} h(\sigma)+\frac{(1-\zeta)\left(1+\zeta^{2}\right) \sigma}{2(\sigma+1)} \frac{\overline{h(\sigma)}}{\sigma-\zeta}\right) \frac{\mathrm{d} \sigma}{\sigma-\zeta} \\
& +\int_{\gamma_{L}} \frac{1}{2 \pi i}\left(\frac{1}{\sigma-\zeta}+(1+\kappa) \frac{(1-\zeta-\sigma) \sigma}{(\sigma-1)\left(\sigma^{2}+1\right)}\right) h(\sigma) \mathrm{d} \sigma \\
& +\int_{-i}^{1}\left(-\frac{\kappa}{2 \pi i} h(\sigma)-\frac{(1-\zeta)\left(1+\zeta^{2}\right) \sigma}{2(\sigma+1)} \frac{\overline{h(\sigma)}}{\sigma-\zeta}\right) \frac{\mathrm{d} \sigma}{\sigma-\zeta} \\
= & (1-\zeta)\left(1+\zeta^{2}\right) F^{\prime}(\zeta),
\end{aligned}
$$

which is considered to be fundamental in the case of zero displacement change boundary conditions.

\section{Numerical Solution of Integral Equations}

We begin with a simple example showing our method of numerical calculation applied in the case of zero displacement change boundary conditions. The Cauchy's integral formula for a function $\chi(\zeta)$, regular inside $\gamma$ and continuous up to its boundary is

Vol. 41, No. 1, 1993 


$$
\frac{1}{2 \pi i} \int_{\gamma} \frac{\chi(\sigma)}{\sigma-\zeta} \mathrm{d} \sigma=\chi(\zeta)
$$

while it is also known that a necessary and sufficient condition for the function $\chi(\sigma)$, continuous on the circle $\gamma$, to be the boundary value of some function, regular inside $\underline{\gamma, \text { is }}(1 / 2 \pi i) \int_{\gamma} \overline{\chi(\sigma)} \mathrm{d} \sigma /(\sigma-\zeta)=\overline{\chi(0)}$ for all $\zeta$ inside $\gamma$ (cf., Muskhelishvili, 1953). Eliminating $\overline{\chi(0)}$ in this equation we may write as

$$
\int_{\gamma} \frac{\overline{\chi(\sigma)}}{\sigma(\sigma-\zeta)} \mathrm{d} \sigma=0
$$

These two Eqs. (26) and (27) can be used to determine numerically the boundary value of $\chi(\sigma)$ from known values of $\chi(\zeta)$ inside $\gamma$, by approximating each integral by summation of unknown values at $N$ points on the circumference of unit circle. That is

$$
\frac{1}{2 \pi i} \sum_{k=1}^{N} \frac{\chi\left(\sigma_{k}\right)}{\sigma_{k}-\zeta_{j}} \Delta \sigma_{k}=\chi\left(\zeta_{j}\right), \quad \sum_{k=1}^{N} \frac{\overline{\chi\left(\sigma_{k}\right)}}{\sigma_{k}\left(\sigma_{k}-\zeta_{j}\right)} \Delta \sigma_{k}=0,
$$

where $\chi\left(\sigma_{k}\right)$ is a set of unknowns on the boundary and $\Delta \sigma_{k}$ is the line element at $\sigma_{k}$ $(k=1,2, \cdots, N)$.

If we have independent linear equations in number $N$ of Eq. (28), we can determine $\chi\left(\sigma_{k}\right)$ for each point on the unit circle. As for such equations, we take in total $N$ equations for distinct $N / 2$ points $\zeta_{j}$ inside $\gamma(j=1,2, \cdots, N / 2)$. We choose those points $\zeta_{j}$ as distributed with equal distance on the circumference of a concentric circle with radius $r_{0}$. For $N=256$ or 512 , we take $r_{0}=0.9$ or 0.95 . Actually separating real and imaginary parts of $\chi(\sigma)$, we deal with a system of $2 N$ linear equations in $2 N$ unknowns.

By solving the system of linear Eq. (28), we can determine $\chi(\sigma)$. This method can be applied even in the case where $\chi(\zeta)$ has logarithmic singularities on the circumference. In Fig. 5, an example of such cases is depicted where $\chi(\zeta)=\ln \left(1+\zeta^{2}\right)$. The boundary value given by this formula is shown in (a) and (b), while numerically calculated one is in (c) and (d). They are in a good agreement. This is for $N=256$ and $r_{0}=0.95$. This method cannot be employed in the case where $\chi(\zeta)$ has a pole on the circumference. In Fig. 6 , the case where $\chi(\zeta)=1 /\left(1+\zeta^{2}\right)$ is depicted. Though the boundary value given by the formula is as in (a) and (b), numerically calculated one is erroneously obtained as in (c).

The case of zero displacement change boundary conditions at $y= \pm H$ is treated by the discretized formulas from Eq. (25) and from equation

$$
\int_{\gamma} \frac{\overline{h(\sigma)}}{\sigma(\sigma-\zeta)} \mathrm{d} \sigma=0 \text {. }
$$

If $h\left(\sigma_{k}\right)$ are successfully determined, we can calculate $h(\zeta)$ at any point $\zeta$ inside $\gamma$ by

$$
h(\zeta)=\frac{1}{2 \pi i} \sum_{k=1}^{N} \frac{h\left(\sigma_{k}\right)}{\sigma_{k}-\zeta} \Delta \sigma_{k}
$$

Table 1 shows the position of $\sigma_{-a}$ for several values of $a / H$. As the ratio $a / H$. 


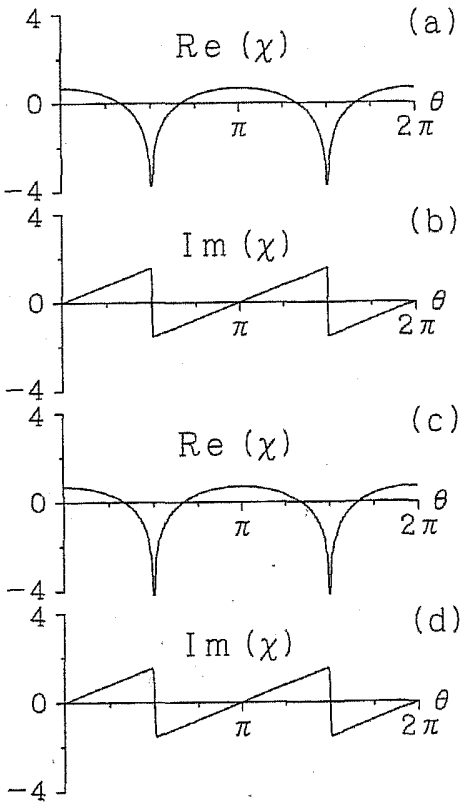

Fig. 5

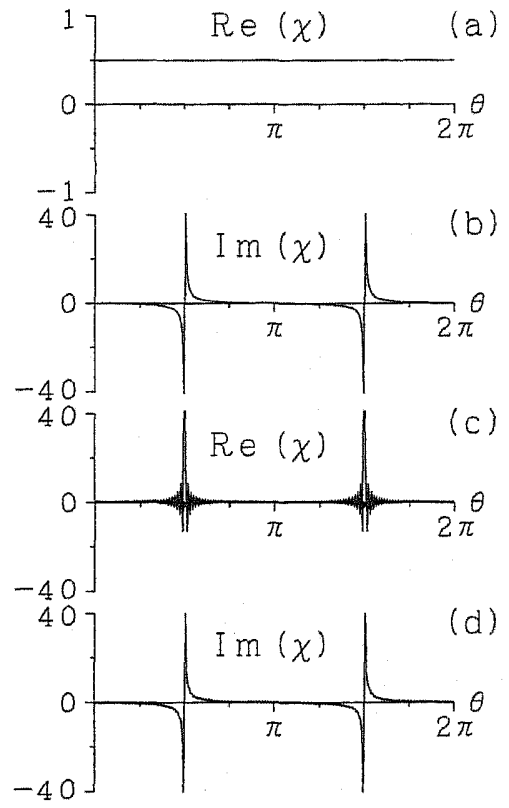

Fig. 6

Fig. 5. (a), (b) Boundary values of $\chi=\ln \left(1+\zeta^{2}\right)$ on the circumference of unit circle. (c), (d) Calculated boundary values by the use of the system of linear equations $\left(N=256, r_{0}=0.95\right)$.

Fig. 6. (a), (b) Boundary values of $\chi=1 /\left(1+\zeta^{2}\right)$ on the circumference of unit circle. (c), (d) Calculated boundary values by the use of the system of linear equations $\left(N=256, r_{0}=0.95\right)$.

Table 1. Position of $\sigma_{-a}\left(\sigma_{-a}=\exp \left(i \theta_{-a}\right)\right)$.

\begin{tabular}{lr}
\hline$a / H$ & \multicolumn{1}{c}{$\theta_{-a}(\mathrm{deg})$} \\
\hline 0.1 & $90+35.12$ \\
0.2 & +21.33 \\
0.3 & +14.00 \\
0.4 & +9.55 \\
0.5 & +6.66 \\
0.8 & +2.42 \\
1.0 & +1.27 \\
2.0 & +0.05 \\
\hline
\end{tabular}

becomes greater, in an approximation of integral by summation of values at discrete $N$ points, a special consideration must be necessary in the vicinity of $\sigma= \pm i$, since in this case the interval between $\sigma=i$, where $g(\sigma)=0$, and $\sigma=\sigma_{-a}$, where $g(\sigma)$ has a logarithmic singularity, may be buried in a single line element $\Delta \sigma_{k}$, the same with the interval between $\sigma=-i$ and $\sigma=\bar{\sigma}_{-a}$.

Vol. 41, No. 1, 1993 


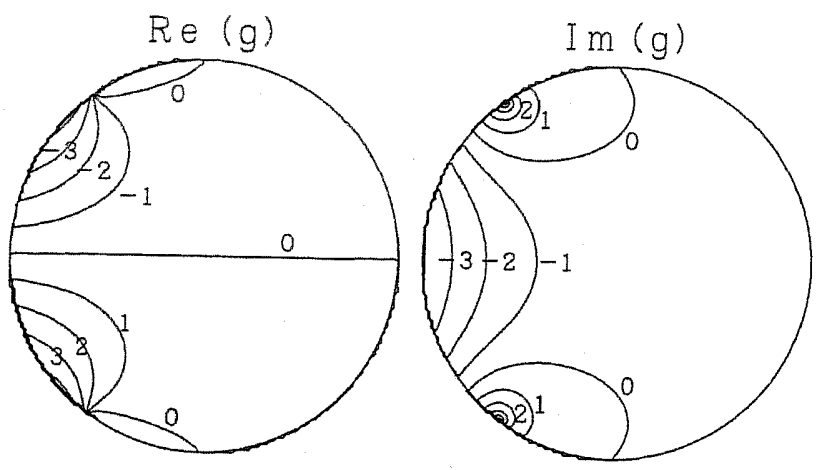

Fig. 7. Contours for $g(\zeta)(a / H=0.1)$.

In our numerical calculations, in obtaining values of $(1-\zeta)\left(1+\zeta^{2}\right) F^{\prime}(\zeta)$ for $\zeta$ inside $\gamma$, we should be careful in choosing proper branches of logarithm included in Eq. (19), in order to keep the regularity of $F^{\prime}(\zeta)$ inside $\gamma$. For this purpose it is helpful to draw contour maps of $F^{\prime}(\zeta)$ inside $\gamma$.

The variation of $(1-\zeta)\left(1+\zeta^{2}\right) F^{\prime}(\zeta)$ inside $\gamma$ is shown in contours for its real and imaginary parts, for $a / H=0.1$ in Fig. 7 , where $g(\zeta)$ is normalized as

$$
\frac{2 \sqrt{\pi}}{\sqrt{\omega^{\prime \prime}(-1)}}(1-\zeta)\left(1+\zeta^{2}\right) F^{\prime}(\zeta)=\left(\tau-\tau_{f}\right) \sqrt{\frac{8 a}{\pi}} g(\zeta) \text {. }
$$

Calculation by Eq. (30) thus gives a smooth curve of $h(\zeta)$ on the real axis and an extrapolation to $\zeta=-1$ yields $\lim _{\zeta \rightarrow-1} h(\zeta)$.

In the case of zero stress change boundary conditions, if we take

$$
h(\zeta)=i(1+\zeta) a_{0},
$$

where $a_{0}$ is an arbitrary real constant, the left side of Eq. (18) automatically vanishes. This shows that in this case the foregoing method applied to the case of zero displacement change boundary conditions may not give a stable solution since the coefficient determinant must vanish at the limit of $N \rightarrow \infty$.

For $h(\zeta)$ we assume that form

$$
h(\zeta)=\sum_{m=0} \alpha_{m} \zeta^{m}, \quad\left(|\zeta| \leq r_{0}<1\right)
$$

then the second term on the left and the right side of Eq. (18) can be written as

$$
\left.\begin{array}{l}
(1-\zeta)\left(1+\zeta^{2}\right) I_{p}^{\prime}(\zeta)=\sum_{m=0} \bar{\alpha}_{m} \sum_{l=0} q_{l m} \zeta^{l}, \\
(1-\zeta)\left(1+\zeta^{2}\right) F^{\prime}(\zeta)=\sum_{m=0} \beta_{m} \zeta^{m},
\end{array}\right\}
$$

respectively. Since $\beta_{m}$ is pure imaginary (as seen in Eq. (19)) and $q_{l m}$ is real (see Appendix 1 ), by putting 


$$
\alpha_{m}=i a_{m}, \quad \beta_{m}=i b_{m},
$$

with real $a_{m}$ and $b_{m}$, we obtain an equation

$$
\sum_{l=0} a_{l} \zeta^{l}-\sum_{l, m=0} a_{m} q_{l m} \zeta^{l}=\sum_{l=0} b_{l} \zeta^{l}
$$

To determine $q_{l m}$ and $b_{l}$ numerically, it is convenient to follow the FFT procedure with respect to the values at $r=r_{0}(<1)$ calculated on the basis of integrals expressed in closed form (Appendix 1). Denoting by $Q_{l m}$ and $B_{l}$ the coefficients then obtained numerically, since $\zeta^{l}=r_{0}^{l} \exp (i l \theta)$, we have

$$
q_{l m}=r_{0}^{-l} Q_{l m}, \quad b_{l}=r_{0}^{-l} B_{l} .
$$

The system of linear equations to be solved is

$$
\sum_{m=0}^{N / 2}\left(r_{0}^{l} \delta_{l m}-Q_{l m}\right) a_{m}=B_{l}, \quad\left(l=0,1, \cdots, \frac{N}{2}\right)
$$

where $\delta_{l m}$ is Kronecker delta. In the matrix on the left side of Eq. (37)

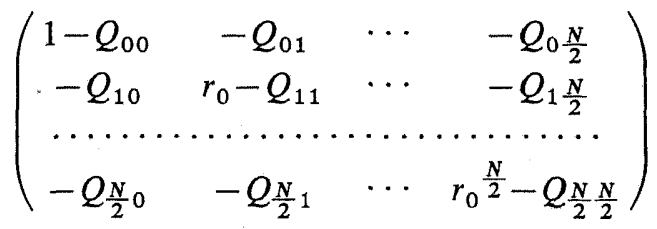

we can verify that the sum of the first and the second column vanishes, which corresponds to the fact that the left-hand side of Eq. (18) vanishes for $h(\zeta)$ as in Eq. (32). Assuming that

$$
\left.h(\zeta)\right|_{\zeta=0}=0 \text {, or } a_{0}=0,
$$

and removing the first column of the matrix, we solve the system of linear equations in $N / 2$ unknowns. Once $a_{m}(m=1,2, \cdots, N / 2)$ is determined we can calculate $h(\zeta)$ at any point $\zeta$ with $|\zeta| \leq r_{0}<1$. From an extrapolation of values of $h(\zeta)$ on the real axis to $\zeta=-1$ we can obtain $\lim _{\zeta \rightarrow-1} h(\zeta)$.

As $H$ tends to $\infty$, we must have the same result irrespective of zero stress and zero displacement change boundary conditions, that is, $K_{I}=0$ and as for $K_{I I}=K_{\tau-\tau_{f}}$

$$
\left(K_{\tau-\tau_{f}}\right)_{H=\infty}=\left(\tau-\tau_{f}\right) \sqrt{\frac{8 a}{\pi}},
$$

which is already known for a homogeneous infinite region (Rice and Simons, 1976).

In Table 2, results of numerical calculations are shown for both boundary conditions, for the ratio $a / H$ less than 1 . Values of $K_{\tau-\tau_{f}}$ are normalized for $\left(K_{\tau-\tau_{f}}\right)_{H=\infty}$. We see in the table that each method of calculation gives stable solutions.

Figure 8 illustrates obtaind values of $K_{\tau-\tau_{f}}$ in the cases of $N=512$ for the zero boundary condition and $N=1,024$ for the zero displacement boundary condition. The ordinate is also normalized for $\left(K_{\tau-\tau_{f}}\right)_{H=\infty}$.

In the case of zero displacement change on the boundary $y= \pm H$, results of

Vol. 41, No. 1, 1993 
Table 2. Table of $K_{\tau-\tau_{f}} /\left(K_{\tau-\tau_{f}}\right)_{H=\infty}$ (Example of results obtained numerically).

\begin{tabular}{|c|c|c|c|c|}
\hline \multirow[b]{2}{*}{$a / H$} & \multicolumn{2}{|c|}{$\begin{array}{l}\text { Case of zero stress change } \\
\text { boundary conditions }\end{array}$} & \multicolumn{2}{|c|}{$\begin{array}{l}\text { Case of zero displacement change } \\
\text { boundary conditions } \\
\qquad(\kappa=2)\end{array}$} \\
\hline & $\begin{array}{c}N=256 \\
\left(r_{0}=0.96\right)\end{array}$ & $\begin{array}{c}N=512 \\
\left(r_{0}=0.98\right)\end{array}$ & $\begin{array}{c}N=512 \\
\left(r_{0}=0.95\right)\end{array}$ & $\begin{array}{l}N=1,024 \\
\left(r_{0}=0.97\right)\end{array}$ \\
\hline 0.1 & 1.047 & 1.052 & 0.975 & 0.976 \\
\hline 0.2 & 1.098 & 1.104 & 0.948 & 0.950 \\
\hline 0.3 & 1.162 & 1.159 & 0.923 & 0.928 \\
\hline 0.4 & 1.200 & 1.209 & 0.899 & 0.907 \\
\hline 0.5 & 1.253 & 1.260 & 0.874 & 0.886 \\
\hline 0.6 & 1.303 & 1.309 & 0.865 & 0.861 \\
\hline 0.7 & 1.378 & 1.357 & 0.840 & 0.836 \\
\hline 0.8 & 1.423 & 1.412 & 0.801 & 0.826 \\
\hline
\end{tabular}
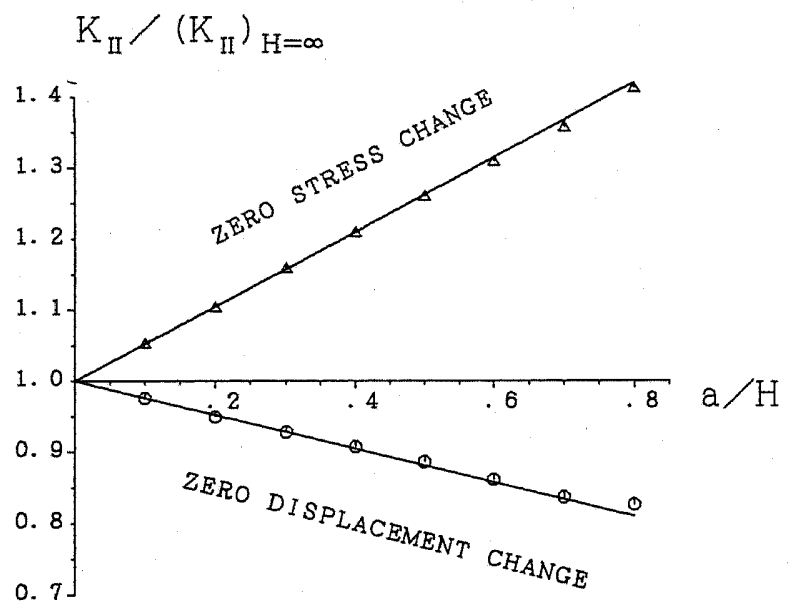

Fig. 8. Normalized stress intensity factors versus $a / H$. Triangles and solid line: case of zero stress change on the parallel boundaries, $K_{\tau-\tau_{f}} /\left(K_{\tau-\tau_{f}}\right)_{H=\infty}=1+$ $k_{s}(a / H)\left(k_{s} \approx 0.52\right)$. Circles and solid line: case of zero displacement change on the parallel boundaries, $K_{\tau-\tau_{f}} /\left(K_{\tau-\tau_{f}}\right)_{H=\infty}=1-k_{d}(a / H)\left(k_{d} \approx 0.24\right.$ for $\left.\kappa=2\right)$.

numerical calculation are shown only for

$$
\kappa=2: \begin{cases}v=1 / 4, & \text { (plane strain) } \\ v=1 / 3, & \text { (plane stress) }\end{cases}
$$

in Table 2 and Fig. 8.

As seen in the figure, the variation in the stress intensity factor against $a / H$ separates in two branches at the point $a / H=0$, according to the two types of boundary conditions. 
For $a / H<1$, obtained stress intensity factor $K_{\tau-\tau_{f}}$ may be expressed in the case of zero stress change boundary conditions, as

$$
K_{\tau-\tau_{f}}=\left(\tau-\tau_{f}\right) \sqrt{\frac{8 a}{\pi}}\left(1+k_{s} \frac{a}{H}\right),
$$

where $k_{s} \approx 0.52$, while in the case of zero displacement change boundary conditions,

$$
K_{\tau-\tau_{f}}=\left(\tau-\tau_{f}\right) \sqrt{\frac{8 a}{\pi}}\left(1-k_{d} \frac{a}{H}\right)
$$

where $k_{d} \approx 0.24$ for $\kappa=2$.

In the case of zero stress change boundary conditions, if we assume $I_{p}(\zeta)=0$ in Eq. (13), as is justified for such small $a / H$ that $\left|\phi^{\prime}(\sigma)\right|$ on $\gamma_{R}$ is negligible in Eq. (14), from $\lim _{\zeta \rightarrow 1} \phi^{\prime}(\zeta)=F^{\prime}(-1)$, we obtain an expression as

$$
K_{\tau-\tau_{f}} \approx \frac{\sqrt{8}\left(\tau-\tau_{f}\right) \sqrt{H}}{\pi} \ln \left[\exp \left(\frac{\pi a}{2 H}\right)+\sqrt{\exp \left(\frac{\pi a}{H}\right)-1}\right] .
$$

The right-hand side is strictly equal to that of Eq. (21) of Fan (1990), though different in appearance. This is a good approximation for a wide range of $a / H$. At first it gives, for $H \rightarrow \infty$, Eq. (39) exactly. For $a / H$ in the range 0 to 1 , with a coefficient $\pi / 12=0.262$ for $k_{s}$ in Eq. (41), the right-hand side of Eq. (43) yields a value which is in the range 1 to 0.84 as large as the result of numerical calculation, and gives, for $a / H \gg 1$,

$$
K_{\tau-\tau_{f}}=\operatorname{const}\left(\tau-\tau_{f}\right) a / \sqrt{H},
$$

where constant is $\sqrt{2}$. Equation (44) agrees with what can be derived by applying path-independent integral (J. R. Rice, personal communication, see Appendix 2).

\section{Construction of a Breakdown Zone Model}

The boundary condition for the problem relating to the effect of the stress $\left(\tau_{b}-\tau_{f}\right)$, shown in Fig. 2, is the one that the third and fourth conditions in Eq. (5) or (21) are replaced by

$$
\left.\begin{array}{l}
y= \pm 0,-\infty<x<-R: \quad \sigma_{y y}=\sigma_{x y}=0, \\
y= \pm 0,-R<x<0: \quad \sigma_{y y}=0, \sigma_{x y}=\tau_{b}-\tau_{f} .
\end{array}\right\}
$$

The corresponding stress intensity factors are obtained as $K_{\tau_{b}-\tau_{f}}$.

Since, at the breakdown zone, the stress should be finite, i.e., there is no stress singularity, the net stress intensity factor must be zero, i.e.,

$$
K=K_{\tau-\tau_{f}}+K_{\tau_{b}-\tau_{f}}=0 .
$$

This determines the breakdown zone size. In the case where $R / H$ is small, the size can be expressed in an explicit form as 


$$
R=\frac{\pi}{8}\left(\frac{K_{\tau-\tau_{f}}}{\tau_{b}-\tau_{f}}\right)^{2}
$$

For a static fault the amount of the slip of the fault at the end of the breakdown zone (i.e., $x=-R, y=0$ ) is

$$
u_{x}(-R,+0)-u_{x}(-R,-0)=\delta=\frac{\left(K_{\tau-\tau_{f}}\right)^{2}}{E^{\prime}\left(\tau_{b}-\tau_{f}\right)}
$$

where

$$
E^{\prime}= \begin{cases}E /\left(1-v^{2}\right), & \text { (plane strain) } \\ E, & \text { (plane stress) }\end{cases}
$$

$E$ being the Young's modulus of the material. In obtaining $\delta$ in Eq. (48) we employed the relation $G=\left(\tau_{b}-\tau_{f}\right) \delta=\left(K_{\tau-\tau_{f}}\right)^{2} / E^{\prime}$, where $G$ is the energy release rate.

According to rupture theory a critical value of $G$ for crack growth is $G=G_{\text {crit }}$, where $G_{\text {crit }}$ is the fracture energy, characteristic of the material. In our case of the simplified relation between the amount of slip and the resistive shear strength, we are concerned with a critical value of $\delta$ denoted by $\delta_{c}$,

$$
\delta=\delta_{c}
$$

such that $G_{\text {crit }}=\left(\tau_{b}-\tau_{f}\right) \delta_{c}$ (cf., Rice and Simons, 1976). If $\delta<\delta_{c}$, the fault is stable, if $\delta=\delta_{c}$, it is in a critical state, while if $\delta>\delta_{c}$, its growth will begin; Eq. (50) is the criterion of the initiation of fault growth.

The critical shear stress $\tau_{c}$ corresponding to the critical state of the fault initiation can be obtained by substitution of Eqs. (48) and (50) into Eq. (41), in the case of zero stress change boundary conditions, as

$$
\tau_{c}-\tau_{f}=\sqrt{\frac{\pi E^{\prime}\left(\tau_{b}-\tau_{f}\right) \delta_{c}}{8 a}} /\left(1+k_{s} \frac{a}{H}\right),
$$

for $a / H<1$. Similarly the critical shear stress $\tau_{c}$ in the case of zero displacement change boundary conditions is given by

$$
\tau_{c}-\tau_{f}=\sqrt{\frac{\pi E^{\prime}\left(\tau_{b}-\tau_{f}\right) \delta_{c}}{8 a}} /\left(1-k_{d} \frac{a}{H}\right),
$$

for $a / H<1$.

\section{Comparison between Two Cases, Discussion and Conclusion}

Equations (51) and (52) show that we have different criterions for two types of boundary conditions. We see in these equations as well as in Eqs. (44) and (1) that the larger is the ratio $a / H$, the greater becomes the discrepancy between the two cases. If we return to our original concept as depicted in Fig. 1(b), both boundary conditions 
of zero stress change and zero displacement change at $y= \pm H$ are merely a simplification of the three-dimensional problem to a two-dimensional one. From the point of original concept, both conditions are to be satisfied at the same time. A question then arises as to either of which condition should be more stressed in our simplification. Though the four curves on the right in Fig. 1(b) represent the displacement before the fracture at the top of the brittle regime, they are taken as corresponding to displacement at the bottom of the brittle regime both before and after the fracture. Thus the condition in displacement will put a restraint to some extent in the upper layer beyond the boundary of the two layers. Further, under zero stress change boundary conditions, a strip will bend freely and displacement perpendicular to the fault is not restricted, while in the case of Fig. 1(b) the strip is surrounded by the medium, restricted in bending. Accordingly the displacement condition is more important. On the other hand, as far as displacement perpendicular to the fault is actually possible above a certain depth, the condition of no change in displacement must be too strong. We may then conclude that what is relevant to our original concept must lie between the two idealized cases. Up to a first approximation based on two-dimensional analyses, as to the critical shear stress for a fault instability, we may suggest the form,

$$
\tau_{c}-\tau_{f}=\sqrt{\frac{\pi E^{\prime}\left(\tau_{b}-\tau_{f}\right) \delta_{c}}{8 a}},
$$

independent of $a / H$. We see in this equation that for fixed $a, \delta_{c}$, and $\tau_{b}-\tau_{f}$, the smaller is $E^{\prime}$, the less is the critical shear stress $\tau_{c}$. On the other hand, using Eqs. (47), (48), and (50), the breakdown size $R$ is expressed in terms of $E^{\prime}, \tau_{b}-\tau_{f}, \delta_{c}$ as

$$
R=\frac{\pi}{8} \frac{E^{\prime} \delta_{c}}{\tau_{b}-\tau_{f}}
$$

independent of $a$.

Equations (53) and (54) are known formulas that have been derived for a homogeneous infinite medium (Rice and Simons, 1976). In that case, $E^{\prime}$ is a material constant to be taken in the infinite medium, that is, at remote points from the fault. But in our case, $E^{\prime}$ is to be taken inside the strip, that is, in the average material including the fault.

The presented breakdown zone model of a fault in a strip is conceptually simple and may be helpful in studying the structural weak line related to a strike slip fault.

We applied linear elasticity theory to a strip. This may be admitted as to obtaining the stress intensity factor for the crack tip.

The authors are grateful to Professor T. Mikumo who encouraged them to prepare the manuscript and to Professor J. R. Rice who not only stimulated an extension of the original wersion of the manuscript but suggested with helpful discussions an important oversight in an extended former version. Permission by Professor Rice to include his communication in the present paper as an appendix is sincerely appreciated. Comments by anonymous reviewers were helpful in improving the manuscript. This work was partly supported by the University of Tokyo 
Fund for the Scientific Research and the National Natural Science Foundation of China. Numerical calculations were carried out by HITAC M-680H at the Earthquake Prediction Data Center, Earthquake Research Institute, the University of Tokyo.

\section{APPENDIX}

\section{Closed Form Integrals for $(1-\zeta)\left(1+\zeta^{2}\right) I_{p}^{\prime}(\zeta)$}

Using the equations

$$
\begin{gathered}
\int \frac{\mathrm{d} \sigma}{\sigma(\sigma-\zeta)}=\zeta^{-1} \ln \left(\frac{\sigma-\zeta}{\sigma}\right) \\
\int \frac{\mathrm{d} \sigma}{\sigma(\sigma-\zeta)^{2}}=-\zeta^{-1} \frac{1}{\sigma-\zeta}-\zeta^{-2} \ln \left(\frac{\sigma-\zeta}{\sigma}\right), \\
\int \frac{\mathrm{d} \sigma}{\sigma^{M}(\sigma-\zeta)}=\sum_{l=1}^{M-1} \frac{1}{M-l} \zeta^{-l} \sigma^{-(M-l)}+\zeta^{-M} \ln \left(\frac{\sigma-\zeta}{\sigma}\right), \quad(M>1) \\
\int \frac{\mathrm{d} \sigma}{\sigma^{M}(\sigma-\zeta)^{2}}=-\sum_{l=1}^{M-1} \frac{l}{M-l} \zeta^{-(l+1)} \sigma^{-(M-l)}-\zeta^{-M} \frac{1}{\sigma-\zeta} \\
-M \zeta^{-(M+1)} \ln \left(\frac{\sigma-\zeta}{\sigma}\right), \quad(M>1)
\end{gathered}
$$

where $M$ is an integer, we obtain closed form expressions for

$$
(1-\zeta)\left(1+\zeta^{2}\right) I_{p}^{\prime}(\zeta)=(1-\zeta)\left(1+\zeta^{2}\right) \frac{1}{2}\left(\int_{1}^{i}+\int_{1}^{-i}\right) \frac{\sigma \overline{h(\sigma)}}{(\sigma+1)(\sigma-\zeta)^{2}} \mathrm{~d} \sigma
$$

If we denote $(1-\zeta)\left(1+\zeta^{2}\right) I_{p}^{\prime}(\zeta)$ for $h(\zeta)=c_{m} \sigma^{m}$ for non-negative integer $m$ by $S_{m}(\zeta)$ we have

$$
\begin{gathered}
S_{0}(\zeta)=\bar{c}_{0}\left\{\frac{1}{2} \frac{(1-\zeta)\left(1+\zeta^{2}\right)}{(1+\zeta)^{2}} \ln \left[1+\left(\frac{1+\zeta}{1-\zeta}\right)^{2}\right]+\zeta\right\} \\
S_{1}(\zeta)=\bar{c}_{1}\left\{-\frac{1}{2} \frac{(1-\zeta)\left(1+\zeta^{2}\right)}{(1+\zeta)^{2}} \ln \left[1+\left(\frac{1+\zeta}{1-\zeta}\right)^{2}\right]+1\right\}, \\
S_{m}(\zeta)=\bar{c}_{m}\left\{\frac { 1 } { 2 } \frac { ( 1 - \zeta ) ( 1 + \zeta ^ { 2 } ) } { ( 1 + \zeta ) ^ { 2 } } \left(\sum_{l=1}^{M-1} \frac{1}{M-l}\left[i^{M-l}+(-i)^{M-l}-2\right]\left[(-1)^{l}-\frac{1}{\zeta^{l}}\right]\right.\right. \\
\left.-\frac{1}{\zeta^{M}} \ln \left[1+\left(\frac{1+\zeta}{1-\zeta}\right)^{2}\right]-\left[(-1)^{M}-\frac{1}{\zeta^{M}}\right] \ln 2\right) \\
(1-\zeta)\left(1+\zeta^{2}\right) \\
\left(\sum_{l=1}^{M-1} \frac{l}{M-l}\left[i^{M-l}+(-i)^{M-l}-2\right] \frac{1}{\zeta^{l+1}}\right.
\end{gathered}
$$




$$
\left.\left.+\frac{M}{\zeta^{M+1}} \ln \left[\frac{1+\zeta^{2}}{\left(1-\zeta^{2}\right.}\right]\right)+\frac{1}{\zeta^{M}}\right\}, \quad(m \geq 2)
$$

where $M=m-1$.

\section{Derivation of Eq. (44)}

Equation (44) can be derived by applying path-independent technique (Rice, 1968) as follows (J. R. Rice, personal communication, 1992). We take the $J$-integral around the following closed circuit (here $l$ and $r$ are introduced, where we let $l \rightarrow \infty, r \rightarrow 0$ ): Begin going anti-clockwise on a small circle of radius $r$ centered on the crack tip at $x=y=0$. Then continue on further contour segments as follows: $x=-r$ to $x=-l$ along $y=+0$ (upper crack surface); $y=0$ to $y=H$ at $x=-l$ (far left of strip); $x=-l$ to $x=l$ at $y=H$ (upper boundary of strip); $y=H$ to $y=-H$ at $x=l$ (far right of strip); $x=l$ to $x=-l$ at $y=-H$ (lower boundary of strip); then from $y=-H$ to $y=0$ at $x=-l$ (far left of strip); and $x=-l$ to $x=-r$ along $y=-0$ (lower crack surface). In the limit $r \rightarrow 0$ the contribution from the first segment is $K_{I I}^{2} / E^{\prime}$. Since $u_{x}(x,+0)=-u_{x}(x,-0)$, from $J=0$ we obtain $K_{I I}=K_{\tau-\tau_{f}}=\left(\tau-\tau_{f}\right) \sqrt{\lambda}$, where $\lambda=2 E^{\prime} u_{x}(-a,+0) /\left(\tau-\tau_{f}\right)$, for both types of boundary conditions. For the problem of zero stress change boundary conditions, when $a / H$ is large, $u_{x}(-a,+0)$ is mainly determined by the stress of type $\sigma_{x x}$ acting over the strip, over the length $a$, where it has an average (from simple equilibrium considerations) of $\left(\tau-\tau_{f}\right) a / 2 H$, and thus produces strain, roughly corresponding $u_{x}(-a,+0) / a$, equal to that average stess divided by $E^{\prime}$. Thus $\lambda$ is roughly $a^{2} / H$, which corresponds (within a numerical factor not far from unity) to Eq. (44).

\section{REFERENCES}

Barenblatt, G. I., The mathematical theory of equilibrium cracks in brittle fracture, Adv. Appl. Mech., 7, 55-129, 1962.

Fan, T. Y., Exact solutions of semi-infinite crack in a strip, Chin. Phys. Lett., 7, 402-405, 1990.

Ida, Y., Cohesive force across the tip of a longitudinal shear crack and Griffith's specific surface energy, J. Geophys. Res., 77, 3796-3805, 1972.

Muskhelishvili, N. I., Some Basic Problems of the Mathematical Theory of Elasticity (transl. by J. R. M. Radok), P. Noordhoff Ltd., Groningen, 1953.

Palmer, A. C. and J. R. Rice, The growth of slip surfaces in the progressive failure of over-consolidated clay, Proc. R. Soc. Lond. A., 332, 527-548, 1973.

Rice, J. R., A path independent integral and the approximate analysis of strain concentration by notches and cracks, Trans. ASME, J. Appl. Mech., 35, 379-386, 1968.

Rice, J. R., The mechanics of earthquake rupture, in Physics of the Earth's Interior, Proc. Int. Sch. Phys. "Enrico Fermi," ed. A. M. Dziewonski and E. Boschi, pp. 555-649, Northholland Publ. Co., Amsterdam, 1980.

Rice, J. R. and D. A. Simons, The stabilization of spreading shear faults by coupled deformation-diffusion effects in a fluid-infiltrated porous materials, J. Geophys. Res., 81, 5322-5334, 1976.

Vol. 41 , No. 1,1993 